\title{
Primärprävention des Schlaganfalls
}

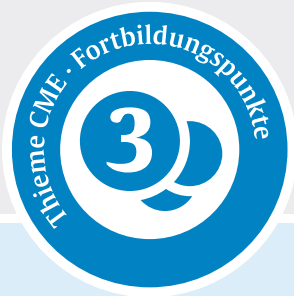

Hans-Christoph Diener ${ }^{1}$, Christian Gerloff ${ }^{2}$

1 Institut für Medizinische Informatik, Biometrie und Epidemiologie (IMIBE), Medizinische Fakultät der Universität Duisburg-Essen

2 Klinik für Neurologie, Universitätsklinikum HamburgEppendorf

\section{ZUSAMMENFASSUNG}

Der Schlaganfall ist eine der Hauptursachen für bleibende Behinderungen. Daher hat die Primärprävention eines ersten Schlaganfalls eine hohe Priorität. Die Primärprävention des ischämischen Schlaganfalls umfasst die Änderung des Lebensstils und der Ernährung, die Behandlung von Risikofaktoren wie Bluthochdruck, Diabetes mellitus und Lipidstörungen, die Thrombozytenaggregationshemmung bei Patienten mit hohem Gefäßrisiko und die Antikoagulation bei Vorhofflimmern (AF). Zu den wichtigsten präventiven Strategien in der Primärprävention von Hirnblutungen gehören die Behandlung von Bluthochdruck, die Reduzierung des Alkoholkonsums und der Verschluss des linken Vorhofohrs bei Patienten mit Vorhofflimmern und dauerhafte Kontraindikationen für eine orale Antikoagulation.

\section{Einleitung}

Es gibt 2 Hauptstrategien im Kampf gegen Schlaganfälle. Erstens, die wirksame Therapie von Patienten mit akutem Schlaganfall, um Tod und Behinderung zu minimieren, zweitens, die Primär- und Sekundärprävention, um Erstund Rezidivereignisse zu verhindern. Diese Übersicht befasst sich mit der Primärprävention von ischämischen und hämorrhagischen Schlaganfällen, also intrazerebralen Blutungen (ICB). Es handelt sich hier um die Aktualisierung einer Übersichtsarbeit aus der Zeitschrift JACC [1]. Patienten mit einem erhöhten Risiko für einen Schlaganfall haben auch ein Risiko für kardiovaskuläre Ereignisse und den vaskulären Tod. Daher gelten die Empfehlungen für die Schlaganfallprävention in den meisten Fällen auch für die Prävention von Myokardinfarkten (MI) und die Behandlung der peripheren arteriellen Verschlusskrankheit (PAD) [2].

Darüber hinaus gibt es 2 Hauptansätze zur Prävention von Schlaganfällen. Zum einen, den „Hochrisiko“-Ansatz, der darauf abzielt, die Prävalenz und das Ausmaß der vaskulären Risikofaktoren bei Hochrisikoindividuen zu reduzieren, und zum anderen, den „Populations (Massen)“-Ansatz, der darauf abzielt, die Prävalenz und das Ausmaß der Risikofaktoren in der Allgemeinbevölkerung zu verringern. Die erste Strategie wird in der Regel von Klinikern und den medizinischen Assistenzberufen umgesetzt und soll daher in dieser Übersicht diskutiert werden. Die zweite Strategie wird in der Regel von Regierungen oder Gesundheitsorganisationen und von Hausärzten, Internisten und Ärzten des öffentlichen Gesundheitswesens umgesetzt.

\section{Primärprävention des ischämischen Schlaganfalls}

Fall-Kontroll-Studien zeigten, dass 10 vaskuläre Risikofaktoren und Begleiterkrankungen $90 \%$ des Schlaganfallrisikos ausmachen [3] ( Tab. 1). Die Bedeutung dieser Risikofaktoren und deren Behandlung wird im Folgenden abgehandelt.

\section{Änderung des Lebensstils}

Zur Prävention des Schlaganfalls wird eine Modifikation des Lebensstils empfohlen, einschließlich gesunder Ernährung [4], Gewichtsverlust, Raucherentwöhnung [5] und regelmäßiger körperlicher Aktivität [6]. Eine systematische Übersicht von randomisierten und kontrollierten Studien (RCTs) von 20 diätetischen Interventionen und Schlaganfallrisiko beobachtete, dass eine Nahrungsergänzung mit Folsäure mit einem niedrigeren Schlaganfallrisiko im Vergleich zu Kontrollen assoziiert war (7 RCTs, 24525 Teilnehmer, 694 Schlaganfallereignisse, relatives Risiko (RR) 0,80; $95 \% \mathrm{Cl}$ : 0,67-0,96). Die Substitution mit einem B-Vitamin-Komplex war mit einem nicht signifikanten Trend zu einem niedrigeren Schlaganfallrisiko im Vergleich zur Kontrolle assoziiert (12 RCTs, 43339 Teilnehmer, 2064 Schlaganfälle, RR 0,90; 0,80-1,01). Eine mediterrane Ernährung war mit einem Trend zu einem niedrigeren Schlaganfallrisiko assoziiert (3 RCTs, 9052 Teilnehmer, 167 Schlaganfälle, RR: 0,65; 0,39-1,11). Die Kombination von Kalzium und Vitamin D war allerdings mit einem erhöhten Schlaganfallrisiko verbunden (7 RCTs, 19227 Teilnehmer, 484 Schlaganfälle, RR 1,17; 1,06-1,30). Eine verringerte Salzaufnahme reduzierte das Risiko der Gesamtmortalität bei Teilnehmern mit arterieller Hypertonie (3 RCTs, 2656 Teilnehmer, 106 Todesfäll, RR, 0,67, 0,46 bis 0,99) 
> Tab. 1 Risikofaktoren für Schlaganfälle in 13447 Fällen eines akuten ersten Schlaganfalls (innerhalb von 5 Tagen nach Symptombeginn) im Vergleich zu 13472 Kontrollen ohne Schlaganfallanamnese, die in der INTERSTROKE-Studie [3] in 32 Ländern zwischen 2007 und 2015 hinsichtlich Alter und Geschlecht verglichen wurden.

\begin{tabular}{|c|c|c|c|c|}
\hline Risikofaktor & $\begin{array}{l}\text { Prävalenz (\%) } \\
\text { bei Kontrollen }\end{array}$ & Odds ratio $(95 \% \mathrm{Cl})$ & Risk ratio & $\begin{array}{l}\text { Populationsbezogenes } \\
\text { Risiko }(99 \% \mathrm{Cl})\end{array}$ \\
\hline Hypertonie oder Blutdruck $\geq 140 / 90$ mmHg & 35 & $3,0(2,7-3,3)$ & 1,54 & $48 \%(45-51)$ \\
\hline Körperliche Aktivität & 16 & $0,6(0,5-0,7)$ & 0,64 & $36 \%(28-45)$ \\
\hline $\begin{array}{l}\text { Apolipoprotein B/A1 Ratio } \\
\text { T2 vs T1 } \\
\text { T3 vs T1 }\end{array}$ & $\begin{array}{l}34 \\
33\end{array}$ & $\begin{array}{l}1,3(1,1-1,4) \\
1,8(1,6-2,1)\end{array}$ & $\begin{array}{l}1,17 \\
1,44\end{array}$ & $\begin{array}{l}- \\
27 \%(22-32)\end{array}$ \\
\hline $\begin{array}{l}\text { Diät (mAHEI Score) } \\
\text { T2 vs T1 } \\
\text { T3 vs T1 }\end{array}$ & $\begin{array}{l}36 \\
30\end{array}$ & $\begin{array}{l}1,35(1,12-1,61) \\
1,35(1,11-1,64)\end{array}$ & $\begin{array}{l}0,84 \\
0,69\end{array}$ & $-\overline{23 \%}(18-29)$ \\
\hline $\begin{array}{l}\text { Waist-to-hip ratio } \\
\text { T2 vs T1 } \\
\text { T3 vs T1 }\end{array}$ & $\begin{array}{l}34 \\
33\end{array}$ & $\begin{array}{l}1,2(1,1-1,4) \\
1,4(1,3-1,6)\end{array}$ & $\begin{array}{l}1,15 \\
1,26\end{array}$ & $\overline{-}$ \\
\hline Psychosoziale Faktoren & 15 & $2,2(1,8-2,7)$ & - & $17 \%(13-23)$ \\
\hline Rauchen & 24 & $1,7(1,5-2,9)$ & 1,45 & $12 \%(10-15)$ \\
\hline Kardiale Ursachen & 5 & $3,2(2,7-3,8)$ & 2,86 & $9 \%(8-10)$ \\
\hline $\begin{array}{l}\text { Alkoholkonsum } \\
\text { gering oder moderat } \\
\text { hoch }\end{array}$ & $\begin{array}{l}25 \\
2,5\end{array}$ & $\begin{array}{l}1,1(1,0-1,39 \\
2,1(1,6-2,7)\end{array}$ & $\begin{array}{l}1,10 \\
2,03\end{array}$ & $\begin{array}{l}- \\
6 \%(3-10)\end{array}$ \\
\hline Diabetes mellitus, $\mathrm{HbA}_{1 \mathrm{c}} \geq 6,5 \%$ & 22 & $1,2(1,1-1,3)$ & 1,12 & $4 \%(2-8)$ \\
\hline Populationsbezogenens Risiko für alle 10 Risikofaktoren & - & - & - & $91 \%(89-92 \%)$ \\
\hline
\end{tabular}

und die Gesamtmortalität bei normotensiven Teilnehmern (3 RCTs, 3518 Teilnehmer, 79 Todesfälle, RR: 0,90, 0,850,95) [7]. Insgesamt ist die Nahrungsergänzung mit Vitaminen und Nahrungsergänzungsstoffen wenig hilfreich.

\section{Bluthochdruck}

Die Senkung des erhöhten Blutdrucks bei Personen mit arterieller Hypertonie ist hochwirksam in der Vorbeugung eines ischämischen Schlaganfalls. Jede Senkung des systolischen Blutdrucks um $10 \mathrm{mmHg}$ und des diastolischen Blutdruck um $5 \mathrm{mmHg}$ reduziert das Risiko eines Schlaganfalls um $41 \%$ (95\% Cl: 33\%-48\%) und kardiale Ereignisse um $22 \%$ (17\%-27\%) ( Tab. 2) [8]. Der systolische Blutdruck sollte vorsichtig auf $<140 / 90 \mathrm{mmHg}$ bei Nichtdiabetikern [8] und $<130 / 80$ mmHg bei Patienten mit Diabetes mellitus gesenkt werden [9]. Die meisten Klassen von blutdrucksenkenden Medikamenten zeigten einen Nutzen bei der Schlaganfallreduktion, mit Ausnahme von Alpha-Blockern [10].

\section{Hyperglykämie und Diabetes mellitus}

Die intensive Behandlung einer Hyperglykämie mit traditionellen Medikamenten bei Patienten mit Diabetes reduziert das Risiko mikrovaskulärer Komplikationen, nicht aber das Risiko eines Schlaganfalls (Hazard ratio (HR) für nicht tödlichen Schlaganfall: 0,96, 0,73-1,27) [11, 12]. Die intensive Therapie zur Senkung des Blutzuckerspiegels hatte keinen signifikanten Einfluss auf die Inzidenz von Schlaganfällen (RR 0,94; 95 \% Cl 0,84-1,06) im Vergleich zur konventionellen Glukosekontrolltherapie nach den Ergebnissen von 13 RCTs mit 58160 Patienten mit Diabetes mellitus Typ 2 [13]. Natrium-Glukose-Kotransporter-2-(SGLT2)-Hemmer verminderten das Risiko für schwerwiegenden vaskuläre Ereignisse, einschließlich Schlaganfall, im Vergleich zu Placebo. Der Haupteffekt wurde aber auf die Inzidenz und das Fortschreiten der Herzinsuffizienz erzielt [14]. Die Gruppe der GLP-Rezeptoragonisten reduziert die Häufigkeit vaskulärer Ereignisse [15]. Eine Reduktion von Schlaganfällen wurde aber nur für Semaglutide beobachtet [16].

\section{Lipidstoffwechselstörungen}

Eine systematische Übersicht, Metaanalyse und Netzwerk-Metaanalyse von RCTs mit Statinen zur Primärprävention kardiovaskulärer Erkrankungen, an der 94283 Personen teilnahmen, zeigte, dass die Statine als Klasse statistisch signifikante Risikoreduktionen bei nicht tödlichem Herzinfarkt (RR 0,62), kardiovaskulärer Mortalität (RR $0,80)$ und nicht tödlichem Schlaganfall $(R R 0,83)$ bewirken [17]. Der absolute Nutzen der Statintherapie hängt vom absoluten Risiko eines Individuums für vaskuläre Ereignisse und der erzielten absoluten Senkung des LDL-Cholesterins ab. Für jede Senkung der LDL-Cholesterinkonzentration um $1 \mathrm{mmol} / \mathrm{L}$ mit einer Statintherapie wird das Risiko eines ersten Schlaganfalls um etwa $21 \%(6 \%-33 \%)$ reduziert. Eine Senkung des LDL-Cholesterins um 2 mmol/L (77 mg/dL) mit einem wirksamen, kostengünstigen Statinschema (z. B. Atorvastatin $40 \mathrm{mg}$ täglich) über 5 Jahre 
- Tab.2 Zusammenfassung der Wirksamkeit von Interventionen zur Primärprävention des ersten Schlaganfalls.

\begin{tabular}{|l|l|l|l|l|}
\hline Intervention & Risk ratio & Schlaganfallrisiko pro Jahr & $\begin{array}{l}\text { Relatives Risiko } \\
\text { (95\% Cl) }\end{array}$ \\
\hline & & Konsolute Risiko- \\
reduktion (ARR)
\end{tabular}

bei 10000 Patienten würde das Auftreten größerer vaskulärer Ereignisse bei etwa 500 Personen (d. h. $5 \%$ absoluter Nutzen), die ein erhöhtes Risiko haben, aber noch kein vaskuläres Ereignis hatten (Primärprävention), auf Kosten der Verursachung symptomatischer unerwünschter Ereignisse (z. B. Muskelschmerzen oder-schwäche) bei bis zu 50-100 Patienten (d. h. 0,5\%-1,0\% absoluter Schaden), Myopathie bei 5 Patienten, neuer Diabetes mellitus bei 50-100 Patienten und möglicherweise ein hämorrhagischer Schlaganfall bei 5-10 Patienten, reduzieren [18]. Unter 39 Studien zur Lipidsenkung, die über ICB bei insgesamt 287651 Teilnehmern berichteten, war die Lipidsenkungstherapie in kombinierten Primär- und Sekundärpräventionsstudien nicht mit einem statistisch signifikanten erhöhten ICB-Risiko verbunden (OR: 1,12; 95 \% Cl 0,981,28) [19]. Der absolute Nutzen der Statine überwiegt eindeutig das Blutungsrisiko bei Personen mit hohem Risiko für schwerwiegende vaskuläre Ereignisse.

Während Statine auch das relative Risiko einer Myopathie erhöhen (RR 1,08), ist das absolute Risiko gering, und insgesamt besteht ein erheblicher Nettonutzen der Statine bei der Prävention schwerwiegender vaskulärer Ereignisse. Unter den Statinen waren Atorvastatin und Rosuvastatin am wirksamsten bei der Reduzierung von Herzinfarkten und Schlaganfällen [18]. Die Statintherapie führt unabhängig vom Alter zu einer signifikanten Reduktion von schwerwiegenden vaskulären Ereignissen, aber es gibt weniger Belege für einen Nutzen in der Primärprävention bei Personen über 75 Jahren, die keine Anzeichen einer Gefäßerkrankung aufweisen [20]. Diese Frage wird in Studien untersucht. Eine Metaanalyse von 20 randomisierten Studien, bei denen zusätzlich zu Statinen monoklonale Antikörper (mAb) gegen PCSK9 (PCSK9-Inhibitoren) bei insgesamt 32329 Teilnehmern und Placebo bei 28549 Teilnehmern eingesetzt wurden, berichtete über eine 23 \%ige (OR 0,77, 0,67-0,89) Verringerung des Schlaganfallrisikos mit Anti-PCSK9 mAb im Vergleich zu Placebo [21] - ohne signifikante Erhöhung des Blutungsrisikos.

Therapien, die primär über eine Hochregulierung der LDL-Rezeptorexpression zur Senkung des LDL-Cholesterins wirken (d. h. Diät, Gallensäure-Sequestriermittel, ile- aler Bypass und Ezetimib), sind mit ähnlichen Risikoreduktionen für vaskuläre Ereignisse pro Veränderung des LDL-Cholesterins assoziiert wie Statintherapien. Die RR für schwerwiegenden vaskuläre Ereignisse pro $1 \mathrm{mmol} / \mathrm{L}$ $(38,7 \mathrm{mg} / \mathrm{dL})$ Reduktion des LDL-Cholesterolspiegels beträgt 0,77 (95\% Cl 0,71-0,84) für Statine und 0,75 (95\% Cl 0,66-0,86) für andere therapeutische Ansätze (Unterschied zwischen den Gruppen, $p=0,72$ ) [22].

\section{Antithrombotische Therapie}

Die Einnahme von Acetylsalicylsäure (ASS) bei Personen ohne kardiovaskuläre Erkrankungen war in 10 randomisierten Studien mit einem geringeren absoluten Risiko eines ischämischen Schlaganfalls verbunden als die Kontrolle (1,27\% ASS vs. $1,48 \%$ ohne ASS, HR: 0,81, $95 \% \mathrm{Cl}: 0,76-$ 0,87, ARR: 0,19\%, $95 \% \mathrm{Cl}: 0,06-0,30)$. Es fand sich aber in 12 randomisierten Studien ein erhöhtes absolutes Risiko für intrakranielle Blutungen $(0,43 \%$ ASS vs. $0,32 \%$ kein ASS, HR 1,34, 1,14-1,57; ARI 0,11\%; 0,04-0,18). In ähnlicher Weise ist die Gabe von ASS bei Personen ohne kardiovaskuläre Erkrankungen mit einem geringeren Risiko für die Kombination von Schlaganfall, MI und kardiovaskulärer Mortalität verbunden (60,2 ASS vs. 65,2 ohne ASS pro 10000 Teilnehmerjahre; ARR 0,41\% (0,23\%-0,59\%; NNT 241). Es besteht aber ein ähnlich erhöhtes Risiko für schwere Blutungen (23,1 ASS vs. 16,4 ohne ASS pro 10000 Teilnehmerjahre; ARI 0,47\% (0,34\%-0,62\%); NNH 210) [23]. Daher kann ASS in der Primärprävention des ischämischen Schlaganfalls nicht empfohlen werden.

Vorhofflimmern ist ein wichtiger Risikofaktor für einen ischämischen Schlaganfall. Die orale Antikoagulation reduziert das Schlaganfallrisiko um 60\%-80\% im Vergleich zu keiner Antikoagulation. In einer Metaanalyse von 4 RCTs, in denen 42411 Teilnehmer mit Vorhofflimmern ein Nicht-Vitamin-K orales Antikoagulans (NOAC) und 29272 Teilnehmer Warfarin erhielten, reduzierten die NOACs das Risiko eines Schlaganfalls oder einer systemischen Embolie (SSE) signifikant um relative 19\% im Vergleich zu Warfarin (absolut 3,1 \% NOAC vs. 3,8\% Warfarin; RR 0,81, $95 \%$ CI 0,73-0,91; ARR 0,7\% über 2 Jahre Follow-up) [24] und reduzierten das Risiko einer intrazerebralen Blutung relativ um 50 \% (absolut 0,44\% NOAC vs. 
0,90\%; RR 0,49, 0,38-0,64; ARR 0,46\%) [24]. Die mediane Zeit im therapeutischen Bereich (INR 2,0-3,0) für Patienten, die Warfarin erhielten wurde, lag zwischen 58\%-68\%. Ein zusätzlicher Nutzen der NOACs besteht darin, dass sie keine regelmäßige Überwachung der Gerinnungshemmung erfordern und keine Wechselwirkung mit der Nahrung aufweisen. Ein weiterer Vorteil ist die Verfügbarkeit spezifischer Gegenmittel [25, 26]. Zu den Einschränkungen der NOACs gehört, dass sie bei Patienten mit fortgeschrittenem Nierenversagen (Kreatinin-Clearance $<15 \mathrm{ml}$ / min) oder bei Dialysepatienten nicht zugelassen sind. ASS [27] und die Kombination von ASS und Clopidogrel [28] haben nur einen begrenzten Nutzen in der Schlaganfallprävention bei Vorhofflimmern und sollten daher nur bei Patienten eingesetzt werden, die eine Antikoagulation verweigern [29]. ASS plus Clopidogrel sind dabei wirksamer als eine ASS-Monotherapie. Der Nutzen wird jedoch durch das erhöhte Blutungsrisiko aufgehoben [30].

\section{Asymptomatische Karotisstenose}

Patienten mit einer asymptomatischen Karotisstenose haben ein sehr geringes Schlaganfallrisiko von weniger als $0,5 \%$ pro Jahr bei einer guten medizinischen Behandlung [31, 32]. Die ursprünglichen randomisierten Studien, bei denen die Karotisendarteriektomie mit einer optimierten medizinischen Behandlung verglichen wurde, wurden zu einer Zeit durchgeführt, zu der keine optimale medizinische Therapie einschließlich Statine zur Verfügung stand [33]. Eine optimale Behandlung der vaskulären Risikofaktoren ist wahrscheinlich genauso wirksam wie die Karotisendarteriektomie oder das Stenting [34, 35]. Eine Registerstudie aus dem VA-System in den USA fand keinen Nutzen einer Operation einer asymptomatischen Karotisstenose im Vergleich zu einer rein konservativen Therapie [36]. Die ACB-Studie (Asymptomatic Cervical Bruit Study) war die einzige, bei der Patienten mit asymptomatischer $>50$ \%iger Karotisstenose, randomisiert wurden und entweder $325 \mathrm{mg}$ ASS täglich ( $\mathrm{n}=188$ ) oder Placebo ( $n=184$ ) erhielten [37]. Nach einer medianen Nachbeobachtung von 2,3 Jahren gab es keine signifikanten Unterschiede in der jährlichen Inzidenz ischämischer Ereignisse (TIA, ischämischer Schlaganfall, instabile Angina pectoris, Herzinfarkt und Tod jedweder Ursache) zwischen den beiden Behandlungen in der ASS- und Placebogruppe (11,0\% vs. $12,3 \%, p=0,61)$. Das Risiko eines ipsilateralen Schlaganfalls allein wurde nicht berichtet. Daher bleibt unklar, ob ASS bei Patienten mit asymptomatischen Karotisstenosen einen Nutzen für die Verhinderung vaskulärer Ereignisse hat [38].

Die COMPASS-Studie verglich niedrig dosiertes Rivaroxaban ( $2 \times 2,5 \mathrm{mg}$ täglich) plus ASS (100 mg täglich), Rivaroxaban ( $1 \times 5$ mg täglich) oder ASS ( $1 \times 100 \mathrm{mg}$ täglich) als Monotherapie bei Patienten mit stabiler koronarer Herzkrankheit und „peripherer arterieller Verschlusskrankheit“ [39]. Eine Untergruppe von Patienten hatte eine asymptomatische Karotisstenose und zeigte einen Trend für einen
Nutzen der Kombinationstherapie gegenüber der Monotherapie [40].

\section{Nicht empfohlene Maßnahmen}

Zu anderen Behandlungsansätzen zur primären Schlaganfallprävention, die keine Wirksamkeit gezeigt haben oder nicht empfohlen werden, gehört der Hormonersatz nach der Menopause [41]. Insgesamt besteht bei oraler Substitution ein leicht erhöhtes Schlaganfallrisiko. Dies gilt nicht für die transdermale Hormonsubstitution mit niedrig dosierten Östrogenpflastern [42]. Der Verschluss eines offenen Foramen ovale bei Personen ohne Schlaganfallanamnese (mit Ausnahme von Berufstauchern) wird ebenfalls nicht empfohlen [43].

\section{Die Polypille}

In Ländern mit niedrigem Einkommen, in denen der Zugang und die Bezahlbarkeit der medizinischen Behandlung möglicherweise eingeschränkt ist, könnte eine Polypille, die ASS, Atorvastatin, Hydrochlorothiazid und entweder Enalapril oder Valsartan enthält, bei Personen mit hohem Risiko einen Schlaganfall verhindern. Eine cluster-randomisierte Studie im Iran beobachtete eine signifikante Verringerung des Risikos eines nicht tödlichen Schlaganfalls (HR 0,44, 0,23-0,82) und eines tödlichen Schlaganfalls (HR 0,38, 0,18-0,82), aber keinen Unterschied in der intrakraniellen Blutung mit einer Polypille im Vergleich zur üblichen medikamentösen Prophylaxe [44].

\section{Primärprävention von Hirnblutungen}

Die wichtigsten Risikofaktoren für intrazerebrale Blutungen (ICB) sind zunehmendes Alter, Bluthochdruck, eine antithrombotische Therapie und eine ausgeprägte Mikroangiopathie, einschließlich der Amylodangiopathie. Von diesen Risikofaktoren sind nur der Bluthochdruck und die Einnahme von antithrombotischen Medikamenten veränderbar. In der PROGRESS-Studie reduzierte die Behandlung mit Perindopril und Indapamid das Risiko einer ersten und wiederkehrenden ICB (HR 0,44 bzw. 0,37) [38]. Es bestand ein nahezu linearer Zusammenhang zwischen der Senkung des Blutdrucks und der Risikoreduktion für Hirnblutungen [45]. Erhöhter Alkoholkonsum und Rauchen sind Risikofaktoren für eine ICB und sollten vermieden werden. Es liegen keine Daten aus randomisierten Studien vor, die belegen, dass ein reduzierter Alkoholkonsum und die Einstellung des Rauchens das Risiko einer ICB verringern.

Wie erwähnt, hat ASS nur eine begrenzte Wirksamkeit in der primären Schlaganfallprävention und birgt ein erhöhtes Risiko für Hirnblutungen sowie für schwere Blutungen. NOACs werden gegenüber VKAs zur Prävention von Schlaganfällen bei Patienten mit Vorhofflimmern empfohlen, vor allen, weil sie das Risiko einer ICB im Vergleich zu VKAs um 50\%-80\% reduzieren (relative Risikoreduktion) [46]. In der AVERROES-Studie hatte Apixaban bei Patienten mit Vorhofflimmern, die sich nicht für Warfarin eigneten, 
ein ähnliches Risiko für ICB wie ASS, war aber bei der Prävention eines ischämischen Schlaganfalls wirksamer [47].

Die Rolle lipidsenkender Medikamente für das Risiko einer ICB wurde in einer kumulativen Metaanalyse von Lipidsenkungsstudien untersucht. Studien mit Statinen, Fibraten, Ezetimib, PCSK9- und CETP-Inhibitoren wurden einbezogen [19]. Bei 39 Studien und 287651 Teilnehmern war die lipidsenkende Therapie in den Studien zur Primärprävention nicht mit einem statistisch signifikant erhöhten Risiko für ICB assoziiert (OR 1,01).

FAZIT

Zusammenfassend lässt sich sagen, dass die Behandlung der Hypertonie die Hauptgrundlage der Therapie zur Senkung des ICH-Risikos ist. ASS sollte nicht zur primären Prävention vaskulärer Ereignisse eingesetzt werden. Bei Patienten mit Vorhofflimmern, die eine Thromboseprophylaxe erfordern, werden NOACs gegenüber VKAs bevorzugt. Lipidsenkende Medikamente sind in der Primärprävention nicht mit einem erhöhten ICB-Risiko verbunden. Die größte Herausforderung ist allerdings die Umsetzung präventiver Maßnahmen in der klinischen Praxis. Viele Menschen im mittleren Lebensalter sind sich ihrer Risikofaktoren nicht bewusst und daher nicht in der Lage, diese zu behandeln. Darüber hinaus ist die Adhärenz zu einer medikamentösen Therapie der arteriellen Hypertonie und der Lipidstoffwechselstörungen leider nicht sehr hoch. Neben der Prävention des Schlaganfalls hat die Behandlung von vaskulären Risikofaktoren darüber hinaus mit hoher Wahrscheinlichkeit einen Einfluss auf die Entwicklung und das Fortschreiten demenzieller Erkrankungen.

Interessenkonflikt

Erklärung zu finanziellen Interessen

Forschungsförderung erhalten: ja; Honorar/geldwerten Vorteil für Referententätigkeit erhalten: ja; Bezahlter Berater/interner Schulungsreferent/Gehaltsempfänger: ja; Patent/Geschäftsanteile/Aktien (Autor/Partner, Ehepartner, Kinder) an Firma (Nicht-Sponsor der Veranstaltung): nein Patent/Geschäftsanteile/Aktien (Autor/Partner, Ehepartner, Kinder) an Firma (Sponsor der Veranstaltung): nein.

Erklärung zu nicht finanziellen Interessen

H.C. Diener hat Forschungsmittel von den folgenden Institutionen erhalten: DFG, BMBF, EU, NIH, EAST-AFnet und Heinz-Nixdorf Stiftung. Er war beteiligt an der Erstellung von Leitlinien der DGN, der DSG, der ESC und EHRA.

\section{Korrespondenzadresse}

\section{Prof. Dr. med. Hans-Christoph Diener}

Institut für Medizinische Informatik, Biometrie und Epidemiologie (IMIBE)

Medizinische Fakultät der Universität Duisburg-Essen Hufelandstr. 55, 45147 Essen

Tel. 0201/7236540

hans.diener@uk-essen.de

\section{Literatur}

[1] Diener HC, Hankey GJ. Primary and Secondary Prevention of Ischemic Stroke and Cerebral Hemorrhage: JACC Focus Seminar. J Am Coll Cardiol 2020; 75(15): 1804-18

[2] Doehner W, Mazighi M, Hofmann BM et al. Cardiovascular care of patients with stroke and high risk of stroke: The need for interdisciplinary action: A consensus report from the European Society of Cardiology Cardiovascular Round Table. Eur J Prev Cardiol 2019: 2047487319873460

[3] O’Donnell M], Chin SL, Rangarajan S et al. Global and regional effects of potentially modifiable risk factors associated with acute stroke in 32 countries (INTERSTROKE): a case-control study. Lancet 2016; 388(10046): 761-75

[4] Dehghan M, Mente A, Teo KK et al. Relationship between healthy diet and risk of cardiovascular disease among patients on drug therapies for secondary prevention. Circulation 2012; 126(23): 2705-12

[5] Duncan MS, Freiberg MS, Greevy RA et al. Association of smoking cessation with subsequent risk of cardiovascular disease. JAMA 2019; 322(7): 642-50

[6] Willey JZ, Moon YP, Paik MC, et al. Physical activity and risk of ischemic stroke in the Northern Manhattan Study. Neurology 2009; 73(21): 1774-9

[7] Khan SU, Khan MU, Riaz $\mathrm{H}$ et al. Effects of nutritional supplements and dietary interventions on cardiovascular outcomes: An umbrella review and evidence map. Ann Intern Med 2019; 171(3): 190-8

[8] Law MR, Morris JK, Wald NJ. Use of blood pressure lowering drugs in the prevention of cardiovascular disease: meta-analysis of 147 randomised trials in the context of expectations from prospective epidemiological studies. BMJ 2009; 338: b1665

[9] Williams B, Mancia G, Spiering W et al. 2018 ESC/ESH Guidelines for the management of arterial hypertension. Eur Heart J 2018; 39(33): 3021-104

[10] The ALLHAT Officers and Coordinators for the ALLHAT Collaborative Research Group. Major cardiovascular events in hypertensive patients randomized to doxazosin versus chlorthalidone. JAMA 2000; 283: 1967-75

[11] Skyler JS, Bergenstal R, Bonow RO et al. Intensive glycemic control and the prevention of cardiovascular events: implications of the ACCORD, ADVANCE, and VA diabetes trials. Circulation 2009; 119(2): 351-7

[12] Reaven PD, Emanuele NV, Wiitala WL et al. Intensive glucose control in patients with type 2 diabetes - 15-year follow-up. N Engl J Med 2019; 380(23): 2215-24

[13] Fang H], Zhou YH, Tian Y] et al. Effects of intensive glucose lowering in treatment of type 2 diabetes mellitus on cardiovascular outcomes: A meta-analysis of data from 58,160 patients in 13 randomized controlled trials. Int J Cardiol 2016; m218: m50-8

[14] Acharya T, Deedwania P. Cardiovascular outcome trials of the newer anti-diabetic medications. Progress in Cardiovascular Diseases 2019; 39(33): 3021-104 
[15] North E], Newman JD. Review of cardiovascular outcomes trials of sodium-glucose cotransporter- 2 inhibitors and glucagon-like peptide- 1 receptor agonists. Curr Opin Cardiol 2019; 34(6): 687-92

[16] Marso SP, Bain SC, Consoli A et al. Semaglutide and Cardiovascular Outcomes in Patients with Type 2 Diabetes. N Engl J Med 2016; 375(19): 1834-44

[17] Yebyo HG, Aschmann HE, Kaufmann M et al. Comparative effectiveness and safety of statins as a class and of specific statins for primary prevention of cardiovascular disease. Am Heart J 2019; 210: 18-28

[18] Collins R, Reith C, Emberson J et al. Interpretation of the evidence for the efficacy and safety of statin therapy. Lancet 2016; 388(10059): 2532-61

[19] Judge C, Ruttledge S, Costello M et al. Lipid Lowering Therapy, Low-Density Lipoprotein Level and Risk of Intracerebral Hemorrhage. J Stroke Cerebrovasc Dis 2019; 28(6): 1703-9

[20] Cholesterol Treatment Trialists Collaboration. Efficacy and safety of statin therapy in older people: a meta-analysis of individual participant data from 28 randomised controlled trials. Lancet 2019; 393(10170): 407-15

[21] Casula M, Olmastroni E, et al. Cardiovascular events with PCSK9 inhibitors: an updated meta-analysis of randomised controlled trials. Pharmacol Res 2019; 143: 143-50

[22] Silverman MG, Ference BA, Im K et al. Association Between Lowering LDL-C and Cardiovascular Risk Reduction Among Different Therapeutic Interventions: A Systematic Review and Meta-analysis. JAMA 2016; 316(12): 1289-97

[23] Zheng SL, Roddick AJ. Association of Aspirin Use for Primary Prevention With Cardiovascular Events and Bleeding Events. JAMA 2019; 321(3): 277-87

[24] Ruff CT, Giugliano RP, Braunwald E et al. Comparison of the efficacy and safety of new oral anticoagulants with warfarin in patients with atrial fibrillation. Lancet 2014; 383(9921): 955-62

[25] Pollack CV, Reilly PA, et al. Idarucizumab for Dabigatran Reversal. N Engl J Med 2017; 377(5): 431-4

[26] Connolly S], Crowther M, Eikelboom JW et al. Full Study Report of Andexanet Alfa for Bleeding Associated with Factor Xa Inhibitors. N Engl J Med 2019; 380: 1326-35

[27] Mant J, Hobbs F, Fletcher $\mathrm{K}$ et al. Warfarin versus aspirin for stroke prevention in an elderly community population with atrial fibrillation (the Birmingham Atrial Fibrillation Treatment of the Aged Study, BAFTA). Lancet 2007: 370(9586): 493-503

[28] Connolly S, Pogue J, Hart R et al. Clopidogrel plus aspirin versus oral anticoagulation for atrial fibrillation in the Atrial fibrillation Clopidogrel Trial with Irbesartan for prevention of Vascular Events (ACTIVE W). Lancet 2006; 367(9526): 1903-12

[29] Steffel J, Verhamme P, et al. The 2018 European Heart Rhythm Association Practical Guide on the use of non-vitamin $\mathrm{K}$ antagonist oral anticoagulants in patients with atrial fibrillation. Europace 2018; 20(8): 1231-42

[30] Connolly S], Pogue J, Hart RG, et al. Effect of clopidogrel added to aspirin in patients with atrial fibrillation. $N$ Engl J Med 2009; 360(20): 2066-78

[31] Marquardt L, Geraghty OC, Mehta Z et al. Low risk of ipsilateral stroke in patients with asymptomatic carotid stenosis on best medical treatment: a prospective, population-based study. Stroke 2010; 41(1): e11-7

[32] Galyfos G, Sachsamanis G, et al. Carotid Endarterectomy versus Carotid Stenting or Best Medical Treatment in Asymptomatic Patients with Significant Carotid Stenosis: A meta-analysis. Cardiovasc Revasc Med 2019; 20(5): 413-23
[33] Raman G, Moorthy D, Hadar N et al. Management strategies for asymptomatic carotid stenosis: a systematic review and meta-analysis. Ann Intern Med 2013; 158(9): 676-85

[34] Reiff T, Eckstein HH, Mansmann U et al. Angioplasty in asymptomatic carotid artery stenosis vs. endarterectomy compared to best medical treatment: One-year interim results of SPACE-2. Int J Stroke 2019: 1747493019833017

[35] Gaba K, Ringleb PA, Halliday A. Asymptomatic Carotid Stenosis: Intervention or Best Medical Therapy? Curr Neurol Neurosci Rep 2018; 18(11): 80

[36] Keyhani S, Cheng EM, Hoggatt KJ et al. Comparative Effectiveness of Carotid Endarterectomy vs Initial Medical Therapy in Patients With Asymptomatic Carotid Stenosis. JAMA Neurol 2020. doi: 10.1001/jamaneurol.2020.1427

[37] Mackey AE, Abrahamowicz M, Langlois Y et al. Outcome of asymptomatic patients with carotid disease. Asymptomatic Cervical Bruit Study Group. Neurology 1997; 48(4): 896-903

[38] Murphy SJX, Naylor AR, Ricco JB et al. Optimal Antiplatelet Therapy in Moderate to Severe Asymptomatic and Symptomatic Carotid Stenosis: A Comprehensive Review of the Literature. Eur J Vasc Endovasc Surg 2019; 57(2): 199-211

[39] Eikelboom JW, Connolly S], Bosch J et al. Rivaroxaban with or without Aspirin in Stable Cardiovascular Disease. N Engl J Med 2017; 377(14): 1319-30

[40] Perera KS, Ng KKH, Nayar S et al. Association Between Low-Dose Rivaroxaban With or Without Aspirin and Ischemic Stroke Subtypes: A Secondary Analysis of the COMPASS Trial. JAMA Neurol 2020; 77(1): 43-48

[41] Yang D, Li J, Yuan Z, Liu X. Effect of hormone replacement therapy on cardiovascular outcomes: a meta-analysis of randomized controlled trials. PloS one 2013; 8(5): e62329

[42] Renoux C, Dell'aniello S, Garbe E, et al. Transdermal and oral hormone replacement therapy and the risk of stroke: a nested case-control study. BMJ 2010; 340: c2519

[43] Pristipino C, Sievert H, D'Ascenzo F et al. European position paper on the management of patients with patent foramen ovale. General approach and left circulation thromboembolism. Eurolntervention 2019; 14(13): 1389-402

[44] Roshandel G, Khoshnia M, Poustchi H et al. Effectiveness of polypill for primary and secondary prevention of cardiovascular diseases (Polylran): a pragmatic, cluster-randomised trial. Lancet 2019; 394(10199): 672-83

[45] The PROGRESS Collaborative Group. Effects of blood pressure lowering with perindopril and indapamide therapy on dementia and cognitive decline in patients with cerebrovascular disease. Arch Intern Med 2003; 163: 1069-75

[46] Kirchhof P, Benussi S, Kotecha D et al. 2016 ESC Guidelines for the management of atrial fibrillation developed in collaboration with EACTS: The Task Force for the management of atrial fibrillation of the ESC Developed with the special contribution of the EHRA of the ESC Endorsed by the ESO. Eur Heart J 2016; 37(39): 2893-2962

[47] Connolly S], Eikelboom J, Joyner C et al. Apixaban in patients with atrial fibrillation. N Engl J Med 2011; 364(9): 806-17

Bibliografie

DOI https://doi.org/10.1055/a-1231-1278

Nervenheilkunde 2020; 39: 636-641

(c) 2020. Thieme. All rights reserved.

Georg Thieme Verlag KG, Rüdigerstraße 14,

70469 Stuttgart, Germany

ISSN 0722-1541 


\section{Punkte sammeln auf CME.thieme.de}

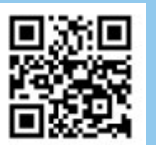

Diese Fortbildungseinheit ist bis zu 12 Monate online für die Teilnahme verfügbar.

Den genauen Einsendeschluss finden Sie beim Modul auf https://cme.thieme.de/CXFH9XI.

Sollten Sie Fragen zur Online-Teilnahme haben, finden Sie unter https://cme.thieme.de/hilfe

eine ausführliche Anleitung. Wir wünschen viel Erfolg beim Beantworten

der Fragen!

Unter https://eref.thieme.de/CXFH9XI oder über den QR-Code kommen Sie

direkt zum Artikel.

VNR 2760512020158722328

\section{Frage 1}

Welches der folgenden Vitamine und Nahrungsergänzungsstoffe reduziert das Schlaganfallrisiko?
A Folsäure
B Vitamin $B_{2}$
C Vitamin $\mathrm{B}_{12}$
D Vitamin D
E Kalzium

\section{Frage 2}

Welche der folgenden Aussagen zur Rolle der arteriellen Hypertonie in der Schlaganfallprävention ist falsch?

A Der Zielblutdruck bei arterieller Hypertonie sollte bei $<140 / 90 \mathrm{mmHg}$ liegen.

B Der Zielblutdruck bei Patienten mit Diabetes mellitus sollte bei< $130 / 80 \mathrm{mmHg}$ liegen.

C Die Kombination von Antihypertensiva ist wirksamer als eine Monotherapie.

D Alpha-Blocker eignen sich besonders zur Primärprävention des Schlaganfalls bei arterieller Hypertonie.

E Bei Patienten mit hochgradigen Stenosen der hirnversorgenden Arterien sollte der Blutdruck vorsichtig gesenkt werden.

\section{Frage 3}

Welche der folgenden Aussagen zur Rolle des Diabetes mellitus und der Hyperglykämie in der Primärprävention des Schlaganfalls ist falsch?

A Metformin und Insulin reduzieren das Risiko mikrovaskulärer Komplikationen.

B Metformin und Insulin reduzieren nicht das Risiko von Schlaganfällen und Herzinfarkten.

C GLP1-Rezeptoragonisten reduzieren die Häufigkeit vaskulärer Ereignisse.

D Eine aggressive Therapie des Diabetes mellitus reduziert das Schlaganfallrisiko.

E Semiglutide reduziert das Risiko für Schlaganfälle.

\section{Frage 4}

Welche der folgenden Aussagen zur Behandlung von Lipidstoffwechselstörungen in der Primärprävention des Schlaganfalls ist falsch?

A Statine reduzieren das Risiko eines Myokardinfarktes.

B Statine reduzieren das Risiko eines nicht tödlichen Schlaganfalls.

C Die Risikoreduktion für den Schlaganfall hängt von dem Serumspiegel des LDL-Cholesterins ab.

D PCSK9-Inhibitoren erhöhen das Risiko intrazerebraler Blutungen.

E Statine können zu Muskelschmerzen und einer Myopathie führen.

\section{Frage 5}

Welche der folgenden Aussagen zur Rolle von Thrombozytenaggregationshemmern in der Primärprävention des Schlaganfalls ist richtig?

A Acetylsalicylsäure reduziert das Risiko von Schlaganfällen in der Primärprävention.

B Acetylsalicylsäure geht in der Primärprävention des Schlaganfalls nicht mit einem erhöhten Blutungsrisiko einher.

C Clopidogrel ist in der Primärprävention des Schlaganfalls wirksam.

D Acetylsalicylsäure hat eine bessere Magenverträglichkeit als Clopidogrel.

E Thrombozytenfunktionshemmer sind in der Primärprävention des Schlaganfalls nicht wirksam und führen zu einem erhöhten Blutungsrisiko.

\section{- Weitere Fragen auf der folgenden Seite ...}




\section{Punkte sammeln auf CME.thieme.de}

Fortsetzung ...

\section{Frage 6}

Welche der folgenden Aussagen zur Primärprävention des Schlaganfalls bei Patienten mit Vorhofflimmern ist falsch?

A Patienten mit Vorhofflimmern haben ein 5-fach höheres Schlaganfallrisiko als Patienten im Sinusrhythmus.

B Vitamin-K-Antagonisten sind in der Primärprävention des Schlaganfalls bei Patienten mit Vorhofflimmern genauso wirksam wie Nicht-Vitamin-K orale Antikoagulantien.

C Der Zielkorridor für die INR in der Primärprävention des Schlaganfalls unter einer Therapie mit Vitamin-K-Antagonisten liegt zwischen 2,0 und 3,0.

D Ab einem INR-Wert von 5,0 steigt das Blutungsrisiko unter einer Therapie mit Vitamin-K-Antagonisten steil an.

E Nicht-Vitamin-K orale Antikoagulantien reduzieren im Vergleich zu Vitamin-K-Antagonisten signifikant das Risiko intrakranieller Blutungen.

\section{Frage 7}

Welche der folgenden Aussagen zu asymptomatischen Karotisstenosen ist falsch?

A Patienten mit asymptomatischen Karotisstenosen haben ein deutlich erhöhtes Schlaganfallrisiko.

B Eine optimale Behandlung vaskulärer Risikofaktoren ist wahrscheinlich genauso wirksam wie eine Karotisendarteriektomie.

C In der Prophylaxe des Schlaganfalls bei Patienten mit asymptomatischen Karotisstenosen ist die Operation genauso wirksam wie das Stenting.

D Bisher ist unbekannt, ob Acetylsalicylsäure bei Patienten mit asymptomatischen Karotisstenosen einen therapeutischen Nutzen hat.

E Bei Patienten mit asymptomatischen Karotisstenosen ist möglicherweise die Kombination von niedrig dosiertem Rivaroxaban plus Aspirin wirksamer als eine Aspirin-Monotherapie.

\section{Frage 8}

Welche der folgenden Aussagen zur Primärprävention des Schlaganfalls ist richtig?

A Bei Frauen sollte nach der Menopause eine Hormonersatztherapie zur Schlaganfallprophylaxe durchgeführt werden.

B Beim offenen Foramen ovale ist der Verschluss mittels eines interventionellen Verfahrens zur Schlaganfallprävention wirksam.

C Ausdauersport hat keinen Einfluss auf das Schlaganfallrisiko.

D Arteriosklerotische Plaques in den Karotiden sind nicht mit einer koronaren Herzerkrankung assoziiert.

E Die Polypille, die ein Statin, Acetylsalicylsäure und Antihypertensiva enthält, ist möglicherweise in der Primärprävention des Schlaganfalls wirksam.

\section{Frage 9}

Welche der folgenden Risikofaktoren spielt die wichtigste Rolle für das Risiko einer intrazerebralen Blutung?
A Arterielle Hypertonie
B Hypercholesterinämie
C Diabetes mellitus
D Niereninsuffizienz
E Alkoholmissbrauch

\section{Frage 10}

Welche der folgenden Aussagen für das Risiko einer intrazerebralen Blutung ist falsch?

A Eine konsequente antihypertensive Therapie reduziert das Risiko von Hirnblutungen.

B Acetylsalicylsäure erhöht das Risiko intrazerebraler Blutungen.

C Apixaban hat ein signifikant höheres Risiko für intrazerebrale Blutungen verglichen mit Acetylsalicylsäure.

D In Metaanalysen haben Statine kein erhöhtes Risiko für intrazerebrale Blutungen gezeigt.

E Reduzierter Alkoholkonsum senkt das Risiko für intrazerebrale Blutungen. 